\title{
An ODE Model of the Motion of Pelagic Fish
}

\author{
Björn Birnir \\ Center for Complex and Nonlinear Science \\ and \\ Department of Mathematics \\ University of California, Santa Barbara
}

January 15,2007

\begin{abstract}
A system of ordinary differential equations (ODEs) is derived from a discrete system of Vicsek, Czirók et al. [35], describing the motion of a school of fish. Classes of linear and stationary solutions of the ODEs are found and their stability explored using equivariant bifurcation theory. The existence of periodic and toroidal solutions is also proven under deterministic perturbations and structurally stable heteroclinic connections are found. Applications of the model to the migration of the capelin, a pelagic fish that undertakes an extensive migration in the North Atlantic, are discussed and simulation of the ODEs presented.
\end{abstract}

\section{Introduction}

The internal dynamics of a school of fish and the migration of the school as a whole present a fascinating problem with many applications. The individual fish tend to adjust their direction and speed to the direction and speed of the school as a whole, see Partridge [28], but the internal structure of the school can be very complicated. We discuss the biology that the model is based on in Section 2. In this paper we show how the commonly used discrete models introduced by Vicsek, Czirók, Ben-Jacob, Cohen, and Shochet in 1995 [35, 15, 13, 14, 36] for the motion of individuals can be turned into ordinary differential equations (ODEs). The ODEs have many advantages over the discrete models: unphysical aspects of some discrete models such as instantaneous turning are eliminated and other aspects such as turning rates are automatically taken care of. It is relatively simple to find solutions of the ODEs and examine their stability. The modern theory of dynamical systems can frequently be used to give a complete qualitative analysis of the phase space of the ODEs thus leading to a complete understanding of the possible solutions. Dynamical systems theory applies to the discrete model as well but in general it is much harder to get concrete results for discrete dynamical systems than continuous.

A review of papers on the Czirók, Vicsek (CV) model and the associated continuum equations, is presented in [16]. There the analogy with models in statistical mechanics is illustrated. The 
CV model possesses both a disordered and an ordered phase, characterized by an order parameter, depending on the level of noise in the system. The other important parameter is the density of the particles. It is shown that when the noise is scaled by the density to the right power, a scaling of the order parameter emerges, close to the critical noise level marking the boundary between ordered and disordered phases. This scaling is similar to the scaling close to a critical temperature in statistical mechanics. Mean field theory is used to derive a continuum equation for the evolution of the density and a hydrodynamic equation is also presented that has vortex solutions. A model studied by Toner and Tu $[34,33]$ determining long range interaction in the continuum is discussed and the associated scalings of the correlations between two location. The scalings of these correlations are shown to be anisotropic (affine), with different exponents along and perpendicular to main motion in an ordered phase.

The work on the CV models clearly shows how methods from statistical mechanics can be used to analyze the case of a large number of particles. It is still essential to study models that can be more closely related to biology and whose simulations can be compared to available data. One such case is the spatial distributions and feeding and spawning migrations of pelagic fish.

The role of the capelin (Mallotus villosusos) in the ecosystem in the arctic ocean around and north of Iceland is discussed in Vilhjálmsson [38] and the assessment of the stock using several surveys each year are discussed by Vilhjálmsson and Carsadden [39]. Following Vilhjálmsson [37, 38] we give a short description of the biology of the capelin and its feeding and spawning migrations that cover hundreds of kilometers in the span of one year.

The capelin in the waters of Iceland and the ocean area between Iceland, Greenland and the island of Jan Mayen is considered to be a separate stock from the existing stocks in the Barent Sea and of Canada. The capelin spawns in shallow water $(10-150 \mathrm{~m})$ of the south and west coast of Iceland. The larvae hatch in approximately 3 weeks after spawning that peaks in March, they drift with the clockwise current around Iceland to the continental shelf north and east of Iceland and to a varying degree to the East Greenland continental plateau. Most juveniles grow up on or in the vicinity of these continental shelves, most of the year's class matures and spawns at age 3 the rest at age 4 . Spawners at age 2 are few and 5-year-old spawners are rare. Maturing capeling age 2 and 3 years (spawning the following year) undertake a migration across the Iceland sea north of Iceland, toward the island Jan Mayen, during spring and summer. The returning migration takes place in September and October and goes east toward Greenland and south into the Denmark straights between Iceland and Greenland, see Figure 14 that shows both the feeding and the spawning migration. These are extensive migrations covering hundreds and sometimes thousands of kilometers. By November the adults have usually assembled near the shelf's edge northwest, north and northeast of Iceland from where another migration, this time a spawning migration, starts in December to January. This migration follows the outer edge of the Icelandic continental shelf in a clockwise direction. In some years a portion of the stock may arrive on the spawning grounds southwest and southeast of Iceland directly from the northwest. However, even in those years most of the stock still follows the traditional route of the spawning migration.

The capelin are pelagic, planktivorous fish and changes in their physical and biological environment profoundly affect their abundance, migration, distribution and growth. They play a key role in the marine ecosystem of this area. They are the single most important item in the diet of the Icelandic cod (Gadus morhua) and several other important commercial fish species in Icelandic and Greenland waters. The capelin is also fed on by marine mammals and seabirds. Because of its importance extensive research and monitoring of the capelin stock, see [39], has taken place since the late 1970 s. 
The ODEs are motivated by applications to pelagic species of fish. In particular we would like to understand the migration route of the capelin in the North Atlantic, see Vilhjálmsson [37, 38]. There already exist both discrete and continuous [4, 24, 25] (partial differential equations) models for this migration. Hubbard, Babak, Magnússon and Sigurdsson [20] added ocean isotherms, food density and directional noise to the CV model in order to induce migration. They also added terms permitting the fish to adjust its speed to the speed of the school. Einarsson, Magnússon and Sigurdsson added ocean currents and the shape of landmasses [26]. They were able to reproduce the capelin migration numerically but at the expense of inserting an artificial bias through the noise. The question one has to answer by such simulations is whether there is a dynamical reason for both the feeding and spawning migration and if so, how they are triggered. The ODEs can be viewed as one step toward answering these questions.

The migration of the capelin is not fundamentally different from the migration of other species of animals. Thus the numerical models and questions asked about other migrations are similar. What are the local interactions between individuals, how does the environment influence the migration, and what makes the animals switch from one migratory phase to another? A fundamental question is what role genetics play. Inherited behavior clearly influences the local interaction but whether it does determine the global features of the migration is an open question. It should be possible to see numerically whether such genetic bias is necessary in order for the models discussed above to produce some of the observed migratory patterns.

Finally the complex configurations (phases) observed in the simulations are intriguing themselves. The individual interactions seem to be able to create very complex behaviors on a global scale and one may ask: Can we use these as models of cells or primitive organisms in biology?

The outline of the paper is as follows. In Section 2 we introduce the discrete model and discuss the experiments and observations of fish schools that justify it. The continuous model is derived in Section 3. It basically follows from the vanishing time-step limit of the discrete model. In Section 4 we find migratory and stationary solutions of the continuous system (ODEs). These solutions are the building blocks from which one hopes to build the migration of the capelin and circling and swarming in the feeding and spawning grounds. In Section 5 we determine the stability of these solutions and discuss how the stationary solutions turn into circling solutions under deterministic perturbations. The perturbations model both the error term separating the discrete system from the ODEs and environmental effects, such as a food and thermal gradients and ocean currents. The stationary solutions turn out to have a very rich structure due to the symmetries of both the discrete and continuous systems and in Section 6 we use equivarient bifurcation theory to find all the stationary solutions with these symmetries. These solutions become periodic orbits when deterministic perturbations are added to the ODEs. Solutions that are periodic orbits under translation of the phase, in polar coordinates, and form tori under deterministic perturbations are found in Section 7. The stability of all of these solutions is explored in Section 8 and structurally stable heteroclinic orbits between stationary solutions are also found. The addition of terms representing attraction and repulsion is discussed in Section 9. In Section 10 we discuss deterministic perturbations of the ODEs and how they can be used to find solutions of the discrete system and predict their stability. In Section 11 applications to the migration of the capelin are discussed and in Section 12 we state our conclusions. Because of the symmetries of the system we write the ODEs in polar coordinates in the paper but in Appendix A the ODEs are written in Cartesian coordinates. 


\section{The Discrete Model}

The two-dimensional discrete model (CV) that was introduced by Vicsek, Czirók, Ben-Jacob, Cohen, and Shochet in 1995 [35] is very simple

$$
\left(\begin{array}{c}
x_{k}(t+\Delta t) \\
y_{k}(t+\Delta t)
\end{array}\right)=\left(\begin{array}{c}
x_{k}(t) \\
y_{k}(t)
\end{array}\right)+v_{k}(t)\left(\begin{array}{c}
\cos \left(\phi_{k}(t)\right) \\
\sin \left(\phi_{k}(t)\right)
\end{array}\right) \Delta t
$$

Here $\left(x_{k}(t), y_{k}(t)\right)$ are the Cartesian coordinates of the $k$ th fish in the plane, at time $t$, and $\left(\cos \left(\phi_{k}(t)\right), \sin \left(\phi_{k}(t)\right)\right)$ is the unit direction vector of its velocity. We will simply call it the direction below. Let $N$ be the number of fish. The fish see other fish and sense each other through their lateral line, either as fluid motion or small pressure variations, see Partridge [28]. This leads to alignment of the individual fish with the direction of the whole school and this can be expressed as

$$
\left(\begin{array}{c}
\cos \left(\phi_{k}(t+\Delta t)\right) \\
\sin \left(\phi_{k}(t+\Delta t)\right)
\end{array}\right)=\frac{1}{N} \sum_{j=1}^{N}\left(\begin{array}{c}
\cos \left(\phi_{j}(t)\right) \\
\sin \left(\phi_{j}(t)\right)
\end{array}\right)
$$

Namely the direction is updated in each time iteration and the new direction of every fish becomes just the average of all the directions. Note that equation (2) is the same for all $k$.

Then the CV model simply says that in every time iteration, a new direction angle is computed, by averaging the directions of all the fish and the position of each fish is updated from its old position by adding the velocity, computed with the new direction, times the length of the time step. This combination of formulas (1) and (2) constitutes the original CV model.

However, there is an important point missing in this iteration which was pointed out by Partridge [28]: when an individual fish comes into a school, it also adjusts its speed to try to travel at the same speed as the other fish. Thus one should also adjust the speeds and this was done by Hubbard, Babak, Magnússon and Sigurdsson [20]. The new speed in each iteration becomes the average speed of the school as a whole

$$
v_{k}(t+\Delta t)=\frac{1}{N} \sum_{j=1}^{N} v_{j}(t)
$$

since the fish try to adjust their speed to that of the school. This completes the discrete model. The directions and speeds are computed in every iteration, using (2) and (3), and the result substituted into (1). This completely determines the trajectory of the school when the initial positions $\left(x_{k}(0), y_{k}(0)\right), 1 \leq k \leq N$ and initial velocities $v_{k}(0)\left(\cos \left(\phi_{k}(0)\right), \sin \left(\phi_{k}(0)\right)\right), 1 \leq k \leq N$ are given, for all the individual fish.

In Partridge and Pitcher [29] the sensory basis is explored for the interaction terms that the (improved) CV model above is based on. It is established in this paper that fish use both their vision and the pressure and fluid velocity variations, sensed by their lateral line, to adjust their direction and speed to that of the other fish in the school. Experiments of blinding the fish and cutting the lateral line, show that both vision and the lateral line provide important and complimentary sensory information. One sense can to some extent compensate for the other but at the cost of changing the relative configurations in the school. The nearest neighbor distance is also increased for the blinded fish whereas it is decreased for the fish with a lateral section. The reaction to the stimulus of these two senses can safely be modeled by a general function of velocities multiplied by a 
function of the directions angles

$$
f\left(v_{1}, \cdots, v_{N}\right) g\left(\phi_{1}, \cdots, \phi_{N}\right)
$$

If we expand $f$ in a general series in the moments (powers) of the velocities and $g$ in a Fourier series of the angles, then the first term can be assumed to have the largest coefficient. Since there is no preferred fish the coefficients of all the velocities in the first moment (the mean) and angles in the first harmonic are one. This is the interaction term in the improved CV model above. Thus the model captures the leading term of the general form of the alignment and speed adjustment. The form of the higher order terms probably depends on the species of fish and would have to be determined by careful experiments. We will discuss the influence of these higher order terms on stability in Section 8. The strength of the interaction combines with the unitless parameter measuring the turning rate. Thus, one can depend on the improved CV model (1-3) for a qualitatively if not quantitatively correct model of the interaction of the individual fish with the school.

Alignment in direction and speed is not the only tendency present in an aggregation of groups of animals. Repulsion when two or more individuals come too close or attraction when two individuals spot each other and move closer are also present [29, 28, 19]. Such models have been presented by Aoki [1], Reynolds [30] and Huth and Wissel [21]. It is straightforward to incorporate such terms in the model and we will do so below, but for the next few sections we concentrate on the alignment and consider repulsion and attraction to be forces that only come into play when the fish are about to collide or are very dispersed and will move toward each other.

\section{The Continuous Model}

Consider the discrete equation (1) written in a form that includes (2) and (3)

$$
\left(\begin{array}{c}
x_{k}(t+\Delta t) \\
y_{k}(t+\Delta t)
\end{array}\right)-\left(\begin{array}{c}
x_{k}(t) \\
y_{k}(t)
\end{array}\right)=\frac{1}{N^{2}} \sum_{j=1}^{N} v_{j}(t-\Delta t) \sum_{j=1}^{N}\left(\begin{array}{c}
\cos \left(\phi_{j}(t-\Delta t)\right) \\
\sin \left(\phi_{j}(t-\Delta t)\right)
\end{array}\right) \Delta t
$$

We divide by $\Delta t$ and send $\Delta t$ to 0 to obtain the ODEs

$$
\left(\begin{array}{c}
\dot{x}_{k}(t) \\
\dot{y}_{k}(t)
\end{array}\right)=\frac{1}{N^{2}} \sum_{j=1}^{N} v_{j}(t) \sum_{j=1}^{N}\left(\begin{array}{c}
\cos \left(\phi_{j}(t)\right) \\
\sin \left(\phi_{j}(t)\right)
\end{array}\right)
$$

\subsection{Polar coordinates}

Introducing complex variables

$$
z_{k}=x_{k}+i y_{k}
$$

we have in polar coordinates

$$
z_{k}=r_{k} e^{i \theta_{k}}
$$

The velocity vector $\dot{z}_{k}=\dot{x}_{k}+i \dot{y}_{k}$ associated to the complex position $z_{k}$ can be written in polar coordinates as

$$
\dot{z}_{k}=v_{k} e^{i \phi_{k}}
$$

where $\phi_{k}$ is the angle of the velocity vector and $v_{k}$ is the speed. 
Differentiating (7) with respect to $t$ gives

$$
\begin{aligned}
\dot{z}_{k} & =\dot{r}_{k} e^{i \theta_{k}}+i r_{k} \dot{\theta}_{k} e^{i \theta_{k}} \\
& =\left(\dot{r}_{k}+i r_{k} \dot{\theta}_{k}\right) e^{i \theta_{k}}
\end{aligned}
$$

and (8) and (9) combine to give

$$
\dot{r}_{k}+i r_{k} \dot{\theta}_{k}=v_{k} e^{i\left(\phi_{k}-\theta_{k}\right)}
$$

By comparing real and imaginary parts in the last equation, we get the two equations

$$
\begin{gathered}
\dot{r}_{k}=v_{k} \cos \left(\phi_{k}-\theta_{k}\right) \\
r_{k} \dot{\theta}_{k}=v_{k} \sin \left(\phi_{k}-\theta_{k}\right)
\end{gathered}
$$

for $k=1, \ldots, N$.

\subsection{The ODE's in polar coordinates}

We now let $\Delta t$ be the discrete time step and let $z_{k}^{n}, \dot{z}_{k}^{n}$ be the $n$-th iterations of the position and the velocity, then

$$
\begin{aligned}
& z_{k}^{n}=r_{k}^{n} e^{i \theta_{k}^{n}} \\
& \dot{z}_{k}^{n}=v_{k}^{n} e^{i \phi_{k}^{n}}
\end{aligned}
$$

Looking at equation (6) we want the next time iterate to be

$$
\dot{z}_{k}^{n+1}=\frac{1}{N^{2}} \sum_{j=1}^{N} v_{j}^{n} \sum_{j=1}^{N} e^{i \phi_{j}^{n}}
$$

Now, let

$$
\zeta=\frac{1}{N^{2}} \sum_{j=1}^{N} v_{j}^{n} \sum_{j=1}^{N} e^{i \phi_{j}^{n}}
$$

and interpolate between velocity vectors, during the $n$th and $n+1$ th time step,

$$
\dot{z}_{k}^{n+1}=\dot{z}_{k}^{n}(1-\alpha \Delta t)+\alpha \zeta \Delta t
$$

where $\alpha$ is a unitless turning rate. It can also be considered to be a measure of the inertia of the fish. From (16) we get

$$
\dot{z}_{k}^{n+1}-\dot{z}_{k}^{n}=\alpha \Delta t\left(\zeta-\dot{z}_{k}^{n}\right)
$$

and by dividing by $\Delta t$ and letting $\Delta t \longrightarrow 0^{+}$we obtain the ODE's.

$$
\ddot{z}_{k}=\alpha \zeta-\alpha \dot{z}_{k} .
$$

We recall from (15) that as $\Delta t \rightarrow 0^{+}$

$$
\zeta=\frac{1}{N^{2}} \sum_{j=1}^{N} v_{j} \sum_{j=1}^{N} e^{i \phi_{j}}
$$


and by (17) and (18)

$$
\ddot{z}_{k}+\alpha \dot{z}_{k}=\alpha \frac{1}{N^{2}} \sum_{j=1}^{N} v_{j} \sum_{j=1}^{N} e^{i \phi_{j}}
$$

Differentiating (8) with respect to $t$ we obtain

$$
\ddot{z}_{k}=\left(\dot{v}_{k}+i v_{k} \dot{\phi}_{k}\right) e^{i \phi_{k}}
$$

and thus (17) and (20) give

$$
\left(\dot{v}_{k}+i v_{k} \dot{\phi}_{k}+\alpha v_{k}\right) e^{i \phi_{k}}=\frac{\alpha}{N^{2}} \sum_{j=1}^{N} v_{j} \sum_{j=1}^{N} e^{i \phi_{j}}
$$

or

$$
\dot{v}_{k}+\alpha v_{k}+i v_{k} \dot{\phi}_{k}=\frac{\alpha}{N^{2}} \sum_{j=1}^{N} v_{j} \sum_{j=1}^{N} e^{i\left(\phi_{j}-\phi_{k}\right)}
$$

By equating real and imaginary parts in the last equation we get the system of ODEs:

$$
\begin{gathered}
\dot{v}_{k}=\frac{\alpha}{N^{2}} \sum_{j=1}^{N} v_{j} \sum_{j=1}^{N} \cos \left(\phi_{j}-\phi_{k}\right)-\alpha v_{k} \\
v_{k} \dot{\phi}_{k}=\frac{\alpha}{N^{2}} \sum_{j=1}^{N} v_{j} \sum_{j=1}^{N} \sin \left(\phi_{j}-\phi_{k}\right)
\end{gathered}
$$

for $k=1, \ldots, N$. Note that solutions to (21) and (22) need to be found in order to solve (10) and (11).

\subsection{The ODE Initial Value Problem}

Now to solve the initial value problem (IVP) for the motion of the school of fish, we must solve the system

$$
\dot{v}_{k}=\frac{\alpha}{N^{2}} \sum_{j=1}^{N} v_{j} \sum_{j=1}^{N} \cos \left(\phi_{j}-\phi_{k}\right)-\alpha v_{k}
$$

$$
\begin{aligned}
v_{k} \dot{\phi}_{k}= & \frac{\alpha}{N^{2}} \sum_{j=1}^{N} v_{j} \sum_{j=1}^{N} \sin \left(\phi_{j}-\phi_{k}\right) \\
\dot{r}_{k} & =v_{k} \cos \left(\phi_{k}-\theta_{k}\right) \\
r_{k} \dot{\theta}_{k} & =v_{k} \sin \left(\phi_{k}-\theta_{k}\right)
\end{aligned}
$$


That is we must first solve the system (23) with the initial speeds and directions

$$
v_{k}(0)=v_{k}^{0}, \phi_{k}(0)=\phi_{k}^{0}
$$

and then solve the system (24), with the above solutions of (23) inserted and the initial radii and position angles

$$
r_{k}(0)=r_{k}^{0}, \theta_{k}(0)=\theta_{k}^{0}
$$

for $k=1, \ldots, N$, in order to get the positions of the fish and velocities at any time $t$. The solution of the initial value problem (23) and (24) is then

$$
r_{k}(t), \quad \theta_{k}(t), \quad v_{k}(t), \phi_{k}(t)
$$

for for $k=1, \ldots, N$.

We have stated the initial value problem here in polar coordinates because it is the easiest coordinated system in which to investigate solutions and their symmetries. In Appendix A we state the initial value problems in Cartesian coordinates for the reader's convenience.

\section{The Migratory and Stationary Solutions}

Now that we have derived a continuous model from the discrete model, we can apply dynamical systems theory to the continuous model. This allows us to analytically find stationary solutions and bifurcations in this set of solutions to the ODEs and use this analysis to predict the behavior of our discrete model under certain conditions. We also can simulate data using both models and compare the results, leading to a firmer understanding of our model and its advantages and pitfalls.

This continuous model has great potential to give us valuable insights into the behavior of the school of fish. With the help of our continuous model, we have been able to find a linear solution and an infinite sequence of stationary solutions for the system. The linear solution, in which all of the fish move in one direction, is the general behavior of fish when they are in their migratory phase, moving as large schools all together toward either the spawning or feeding grounds. Let the turning rate be $\alpha=1$ for the remainder of the analysis; this is not necessary for the existence of these solutions but this choice of $\alpha$ simplifies the exposition. By analysis of our ODEs (23)-(24), we see that the migratory behavior occurs when the direction angles of all of the fish are identical:

$$
\begin{aligned}
\dot{v}_{k} & =\frac{1}{N^{2}} \sum_{j=1}^{N} v_{j} \sum_{j=1}^{N} \cos \left(\phi_{j}-\phi_{k}\right)-v_{k} \\
& =\frac{1}{N^{2}} \sum_{j=1}^{N} v_{j} \sum_{j=1}^{N} \cos (0)-v_{k} \\
& =\frac{1}{N} \sum_{j=1}^{N} v_{j}-v_{k}=0
\end{aligned}
$$

if

$$
v_{k} \equiv v
$$


for all $k$. Thus, in this case, $v_{k}$ is a constant. Moreover by the same argument the second equation in (23) gives

$$
v \dot{\phi}_{k}=0
$$

so if $v \neq 0$,

$$
\phi_{k} \equiv \phi
$$

is also a constant for all $k$.

Now if we substitute these solutions of the equations (23) into the equations (24) we get the equations

$$
\dot{r}_{k}=v \cos \left(\phi-\theta_{k}\right)
$$

and

$$
r_{k} \dot{\theta}_{k}=v \sin \left(\phi-\theta_{k}\right)
$$

A simple solution of these equations is

$$
\theta_{k}(t)=\theta_{k}(0)=\phi
$$

and

$$
r_{k}(t)=r_{k}(0)+v t
$$

Because this last equation is the equation of a straight line and the slope of the line is $v$ for all $k$, it is clear that the fish will move as a school, all in the same direction $\phi$, with constant speed $v$, on a single line. Indeed, the general asymptotic solution of the initial value problem (23) and (24) of this type can be expressed as

$$
r_{k}=r_{k}^{0}+v t, \theta_{k}=\phi+\frac{r_{k}^{0}}{r_{k}^{0}+v t}\left(\theta_{k}^{0}-\phi\right), \phi_{k}=\phi, v_{k}=v
$$

up to leading order in powers of $\frac{1}{r_{k}^{0}+v t}$, for all $k, 1 \leq k \leq N$. Figure 1 is a school of fish having these same properties (same speed and direction) whose motion is parallel but is not restricted to lie on a single line. (It is, however, asymptotic to a single line.)

In addition to these linear solutions, we find an infinite family of stationary solutions; letting the number of fish be any integer $N$, a stationary solution is attained by choosing the direction vector of the $k$ th fish in our school be the $k$ th power of the (primitive) $N$ th roots of unity $e^{i \phi_{k}}=e^{i \omega_{k}}=$ $e^{k i \omega}, 1 \leq k \leq N$, where

$$
e^{i \omega}=1^{\frac{1}{N}}
$$

To see that these angles produce stationary solutions, consider the system (23) once again:

$$
\begin{aligned}
\dot{v}_{k} & =\frac{1}{N^{2}} \sum_{j=1}^{N} v_{j} \sum_{j=1}^{N} \cos \left(\phi_{j}-\phi_{k}\right)-v_{k} \\
v_{k} \dot{\phi}_{k} & =\frac{1}{N^{2}} \sum_{j=1}^{N} v_{j} \sum_{j \neq k}^{N} \sin \left(\phi_{j}-\phi_{k}\right)
\end{aligned}
$$

Now the sum of all the $N$ th root of unity vanishes

$$
e^{i \omega}+e^{2 i \omega}+\cdots+e^{(N-1) i \omega}+1=0
$$


and multiplying by $e^{-i \omega_{k}}$ and taking the real and imaginary parts, we see that

$$
\sum_{j=1}^{N} \cos \left(\omega_{j}-\omega_{k}\right)=0=\sum_{j \neq k}^{N} \sin \left(\omega_{j}-\omega_{k}\right)
$$

(The easiest way of seeing that the sum of the $N$ th root of unity vanish is to expand the polynomial in the equation

$$
z^{N}-1=0 B
$$

into a product and notice that all the symmetric functions of the roots that are the coefficients in the polynomial vanish except the last one.)

Thus the equation above reduces to the equations

$$
\dot{v}_{k}=-v_{k}, \quad \dot{\phi}_{k}=0
$$

if $v_{k} \neq 0$, which have the solutions

$$
v_{k}=v_{k}^{0} e^{-t}, \phi_{k}=\omega_{k}
$$

Substituting these solutions of the equations (23) into the equations (24) gives us the equations

$$
\begin{gathered}
\dot{r}_{k}=v_{k} \cos \left(\omega_{k}-\theta_{k}\right) \\
r_{k} \dot{\theta}_{k}=v_{k} \sin \left(\omega_{k}-\theta_{k}\right)
\end{gathered}
$$

The solutions of these equations are asymptotically stationary, so it does not matter which initial values $r_{k}$ and $\theta_{k}$ have; $v_{k}$ is decaying exponentially in $t$ and the solution quickly approaches a stationary solution. If we pick the initial angles $\theta_{k}(0)=\omega_{k}$ or distribute the fish at the $N$ roots of unity around the circle we get the solutions

$$
r_{k}=r_{k}^{0}-v_{k}^{0} e^{-t}, \theta_{k}=\omega_{k}
$$

These are clearly asymptotically stationary solutions.

We summarize the (asymptotically) stationary solutions that we have found of the system (23) and (24). They are

$$
r_{k}=r_{k}^{0}-v_{k}^{0} e^{-t}, \theta_{k}=\omega_{k}, v_{k}=v_{k}^{0} e^{-t}, \phi_{k}=\omega_{k}
$$

where $e^{i \omega_{k}}$ is an $N$ th root of unity. Thus, for all positive integers $N$, there is such an asymptotically stationary solution. But as we will see below this is just the tip of the iceberg.

We now have two classes of solutions that may be called linear and stationary, see Figures 1 and 2. We will call the linear ones

\section{Migratory Solutions:}

$$
\begin{aligned}
r_{k}(t) & =r_{k}^{o}+v t \\
\theta(t) & =\phi+\frac{r_{k}^{0}}{r_{k}^{0}+v t}\left(\theta_{k}^{0}-\phi\right) \\
v_{k}(t) & =v \\
\phi_{k}(t) & =\phi
\end{aligned}
$$




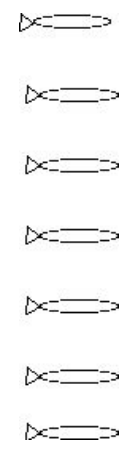

Figure 1: The Migrating School

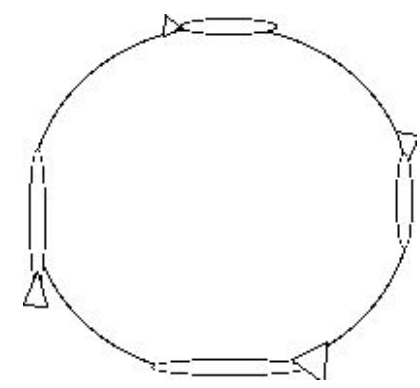

Figure 2: The Stationary School

where $\phi$ is an arbitrary angle determined by the initial condition. These are solutions to the system $(23,24)$ up to leading order in powers of $\frac{1}{r_{k}^{0}+v t}$. The solution (28) is asymptotic to a solution of $(23,24)$ consisting of $N$ chasing each other on a single line. The other class consisting of stationary solutions will accordingly be called

\section{Stationary Solutions:}

(29)

$$
\begin{aligned}
r_{k}(t) & =r_{k}^{o} \\
\theta(t) & =\omega_{k} \\
v_{k}(t) & =0 \\
\phi_{k}(t) & =\omega_{k}
\end{aligned}
$$

These solutions are solutions to the system $(23,24)$. Migratory solutions are simulated in Figure 3 and deterministic perturbations of stationary solutions are simulated in Figures 4 and 5.

Examples of a migratory and stationary solutions are depicted in Figures 1 and 2 respectively. 


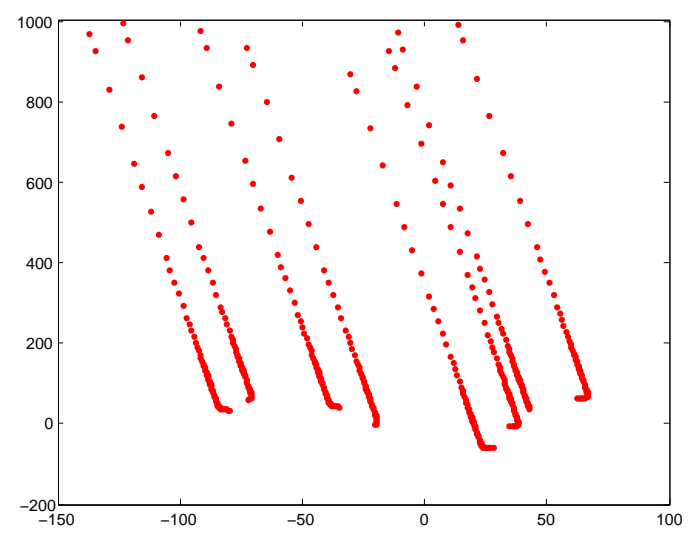

Figure 3: The Migrating Solution of the ODEs. This simulation of the ODEs shows a typical migratory solution in the $x-y$ plane. The eight fish are started with arbitrary positions and velocities but after a very short time they line up. The turning rate is $\alpha=1$, and the dimensionless units would be given the units centimeters in case of the capelin. 


\section{Stability and Deterministic Perturbation}

In this section we perform the stability analysis of the Migratory and Stationary solutions and discuss what kind of behavior of solutions the stability analysis implies in the presence of deterministic perturbations. We will also discuss the rationale for adding such perturbations.

We need to linearize the system (23) and (24) respectively around the Migratory and Stationary solutions and then compute the eigenvalues of the resulting Jacobian matrix. The system (23) does not depend on the $r$ and $\theta$ variables and thus the Jacobian falls into two $2 N \times 2 N$ matrices whose eigenvalues can evaluated separately. Moreover, the latter $2 N \times 2 N$ matrix corresponding to the system (24) splits into $N, 2 \times 2$ matrices, whose eigenvalues are easily found.

\subsection{Stability of Migratory Solutions}

In the migratory case, the Jacobian of the ODE vector field $F(r, \theta)$ (right hand side of the ODEs (23)) linearized about the migratory solutions (28), consists of Toeplitz matrices, see [3], whose eigenvalues and eigenvectors can be explicitly computed. The formulas for the eigenvalues are given in Section 8. Counting eigenvalues, we get $N$ zero eigenvalues corresponding to the $v_{k}$ directions, $N-1$ negative eigenvalues corresponding to the $\phi_{k}$ directions and one zero eigenvalue corresponding to the diagonal $N$-torus $T^{n}$ rotation implemented by changing the common direction angle $\phi$. For the system (24) we are investigating the stability of the linear trajectories $r_{k}=r_{k}^{0}+v t$ that is determined by the angle equation

$$
\dot{\theta}_{k}=\frac{v}{r_{k}} \sin \left(\phi-\theta_{k}\right)
$$

Linearizing this equation about (28) gives

$$
-\frac{v}{r_{k}^{0}+v t} \cos \left(\phi-\theta_{k}\right) \rightarrow-\frac{v}{r_{k}^{0}+v t} \cos \left(\phi-\theta_{k}^{0}\right)<0
$$

for $\pm\left(\phi-\theta_{k}^{0}\right)<\pi / 2$. This produces $N$ negative eigenvalues for every fixed $t$ and thus the Migratory solution is stable except for changes in the speeds $v_{k}$ and the common angle $\phi$.

\subsection{Stability of Stationary Solutions}

A similar analysis of the stationary solution (29) with $\phi_{k}=\omega_{k}$ gives a Jacobian with $N$ zero eigenvalues corresponding to the $v_{k}$ directions but 2 positive eigenvalues and $N-3$ zero eigenvalues, corresponding to the $\phi_{k}$ directions, see Section 8 for details. Then for the system (23) we have to add one positive eigenvalue corresponding to the drift of $\phi$ along the diagonal of all the $\phi_{k} s$. The $2 \times 2$ matrices corresponding to the position coordinates $r_{k}, \theta_{k}$ have two zero eigenvalues. Thus the stationary solution (29) is unstable.

\subsection{Deterministic Perturbations}

Fish are typically not stationary and one may wonder whether the stationary solutions have any biological significance at all. As shown above the $\operatorname{ODE}(23,24)$ system is the continuum limit 


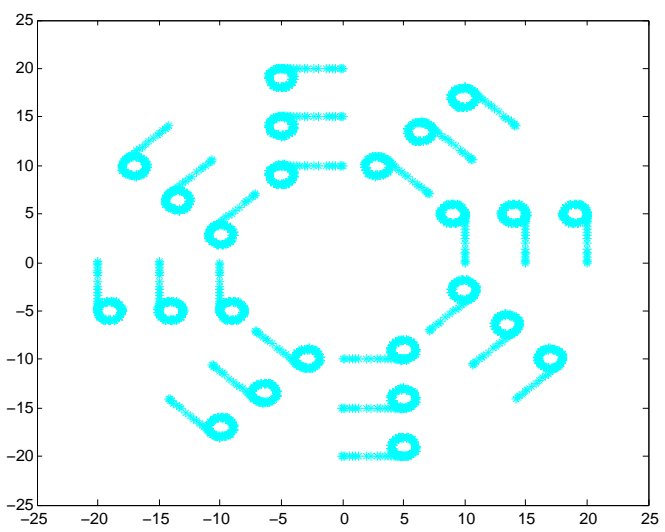

Figure 4: The Circling Schools of Fish, with small velocity. In this simulation the fish are given initial positions and velocities close to their stationary solutions. A deterministic perturbation is added to the equations, see Equations $(35,36)$, the speed perturbation is $v=0.0001$ and the perturbation of the direction angle is $v \omega=0.0001$, the turning rate is $\alpha=1$. 


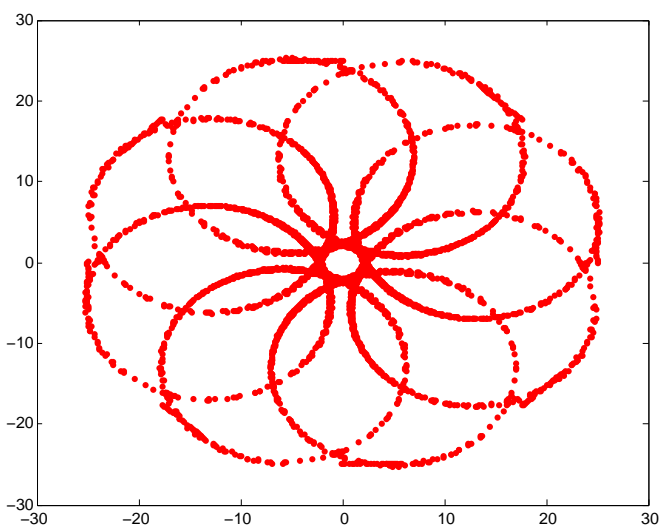

Figure 5: The Circling Schools of Fish, with larger velocity. This simulation of the ODEs is similar to Figure 8, except the perturbation of the speed is larger. A deterministic perturbation is added to the equations, see Equations $(35,36)$, the speed perturbation is $v=0.0001$ and the perturbation of the direction angle is $v \omega=0.0012$, the turning rate is $\alpha=1$. 
of the discrete system. Thus for small time steps we can consider the discrete system to be a deterministic perturbation of the ODEs. In addition, for realistic simulations one wants to include environmental effect such as thermal gradients, currents and food gradients. These can all be simulated as deterministic perturbations. When small perturbations are added to the system (23) the stationary solutions turn into periodic orbits. The radius of these periodic orbit in the $r, \theta$ plane depends on the speed which is the perturbation to the $v_{k}$ equation. Small speeds produce small circles, larger perturbations of the speed large circles. Two such cases are shown in Figures 3 and 4.

The stability of the Migratory solution means that the perturbations do not affect it much unless they are very large. The only thing that can happen is that the velocities change a little as does the common angle (direction) $\phi$ of the school.

The stationary case is much richer and more varied. It possesses symmetries that imply that whole families of stationary solutions exist and all of these families can change into periodic orbits or even quasi-periodic orbits on tori in the presence of deterministic perturbations. To understand these families and their stability we must apply equivariant bifurcation theory, see Golubitsky, Stewart and Schaffer [18].

\section{Equivariant Bifurcation Theory and Stationary Schools}

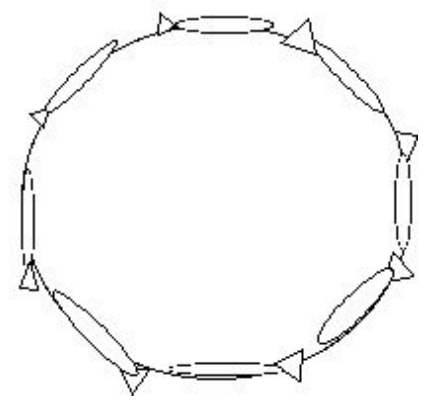

Figure 6: Stationary School Located at the Roots of Unity $m=8, k=1$.

We now apply a theorem in Ashwin and Swift [3] to describe the stationary solutions of the system $(23,24)$. The theorem will give us configurations of schools of fish that are stationary. Then we will add deterministic perturbations and by a similar argument as above show how these lead to solutions that are schools of fish executing a periodic (circling) motion. These configurations are relatively easy to understand so we will describe them first. The theorem requires some mathematical background that we will explain but we refer the mathematically sophisticated reader to the paper by Ashwin and Swift [3] for the details of the proof. The simplest configuration is a group of fish located around a circle as in the Stationary solutions (29) their locations given by the $N$ th roots of unity, see Figure 6 . The next configuration is to give each fish $k-1$ companions located at different radii but at the same angle, see Figure 7 . The total number of fish is now $N=m k$, that is each group of $k$ aligned fish is stationed at a position angle that is an $m$ th root of unity. Next we make $l$ groups at each $m$ th roots of unity. Each group contains $k_{j}$ fish positioned in a radial forma- 


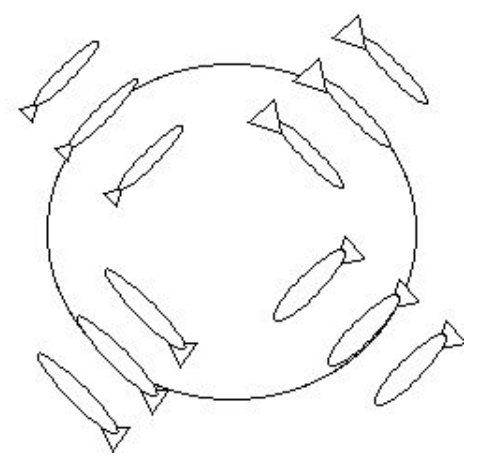

Figure 7: Four Stationary Groups, $m=4, k=3$.

tion, and we have to specify $l-1$ parameters $\delta_{j}$ that give the distance between the $l$ groups, see Figure 7. The total number of fish is $N=\left(k_{1}+\cdots+k_{l}\right) m$. It follows from the theorem below that with repulsion and attraction implemented, see Section 9 so that the fish cannot come too close to each other, or be too far apart, these are all the possible asymptotically stationary solutions. Each configuration is an $l$ torus $T^{l-1} \times T^{1}$, parametrized by the $l-1$ parameters $\delta_{j}, 1 \leq j \leq l-1$ and the rotation angle $\phi$.

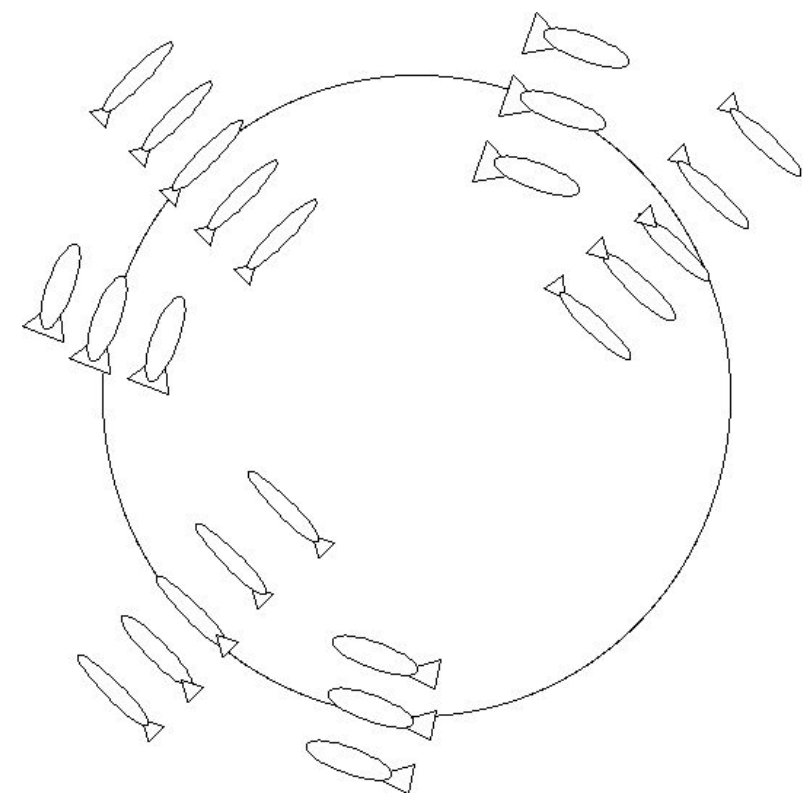

Figure 8: The Stationary Clusters of Schools, $k_{1}=5, k_{2}=3, m=24$.

We will now explain the mathematical background for the theorem that give these above configurations. Golubitsky, Stewart and Schaffer [18] and Brown, Holmes and Moehlis [9] can be consulted for more background. Let

$$
\dot{x}=f(x)
$$

be an ODE on a manifold $M$ and assume that $\Gamma$ is a group acting on $M$. The ODE is $\Gamma$ invariant if $f$ commutes with the group action or $f(\gamma x)=\hat{\gamma} f(x), \gamma \in \Gamma$. Here $\hat{\gamma}$ denotes the derivative map, 
see Arnold [2] which acts on the tangent space $T_{x} M$. The isotropy subgroup $\Sigma_{x_{o}}$ consists of all the group elements that leave the solution $x_{o}$ invariant

$$
\Sigma_{x_{o}}=\left\{\gamma \mid \gamma x_{o}=x_{o}\right\}
$$

Associated with each isotropy subgroup is a subspace of fixed points

$$
\operatorname{Fix}\left[\Sigma_{x_{o}}\right]=\left\{x \in M \mid \gamma x=x \text { for all } \gamma \in \Sigma_{x_{o}}\right\}
$$

It follows immediately that if $x(t)$ is a solution of the ODE then $\gamma x(t)$ is also a solution and the

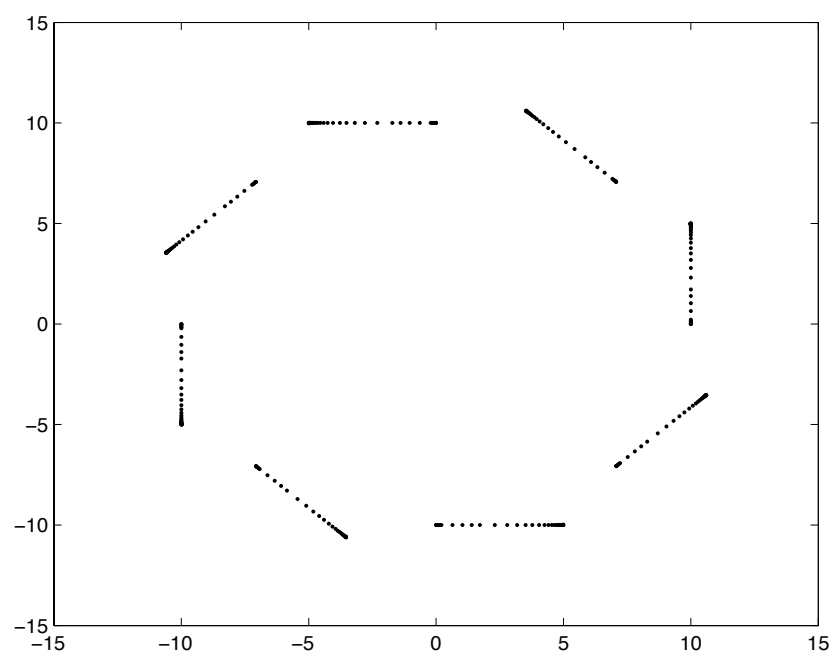

Figure 9: The First Stationary School. This is a simulation of the ODEs finding the stationary solutions in Figure 6. The fish travel in straight lines and stop at the stationary solutions, the turning rate is $\alpha=1$.

subspace of fixed points Fix $\left[\Sigma_{x_{o}}\right]$ is invariant under the flow defined by the ODE. Now we can state the theorem:

Theorem 6.1 (Ashwin and Swift [3]) Every isotropy subgroup of a general $S_{N} \times T^{1}$ equivariant vector field is of the form

$$
\Sigma_{\mathbf{k}, m}=\left(S_{k_{1}} \times \cdots \times S_{k_{l}}\right)^{m} \rtimes Z_{m}
$$

where $N=m\left(k_{1}+\cdots+k_{l}\right)$ and $\rtimes$ denotes the semi-direct product.

The groups are $S_{N}$, the group of permutations of $N$ objects, the action on $S_{N}$ is the interchange of any two objects without regard to order, $T^{1}$ is the circle and the action on $T^{1}$ is just translation mod $2 \pi\{\phi \rightarrow \phi+\psi\}$. This generalizes to the diagonal of the $N$ torus $T^{N}\left\{\phi_{k} \rightarrow \phi_{k}+\psi\right\}$, and the cyclic subgroups $Z_{m}$ of $T^{1}$ with action $\left\{\phi \rightarrow \phi+\frac{2 \pi}{m}\right\} \bmod 2 \pi$. By considering Figure 8 it becomes clear that the action on $S_{k_{j}}$ corresponds to a permutation of the position (and the direction) of fish in the $k_{j}$ group, in each cluster of groups. The action on $Z_{m}$ is just rotating the $i$ th (of $m$ ) cluster into the $i+1$ th (as the lock on a safe) and the $m$ th to the first, and finally the torus action is the translation of the rotation angle $\phi$. 


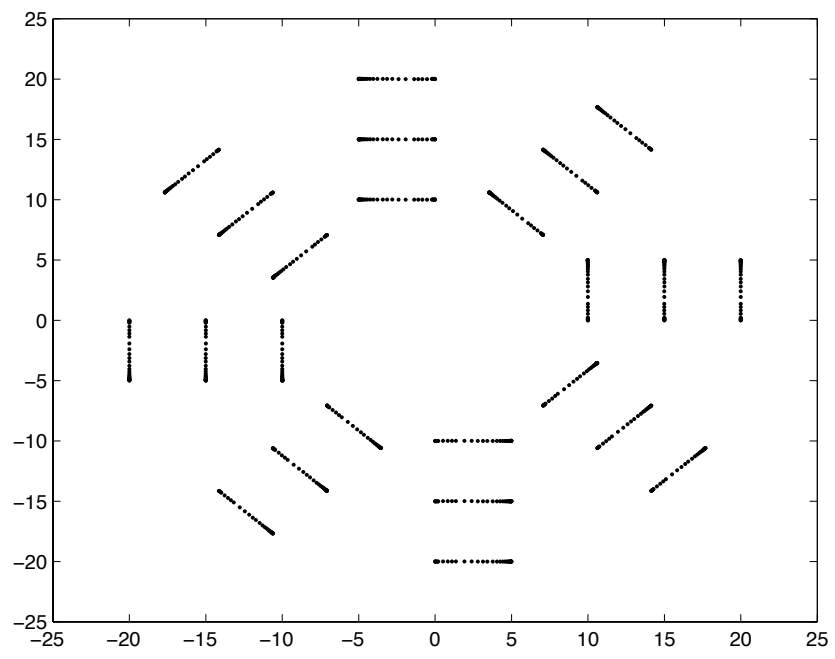

Figure 10: The Second Stationary School. This is a simulation of the ODEs finding the stationary solutions analogous to those in Figure 7. The fish travel in straight lines, in 8 subschools of three fish each, and stop at the stationary solutions, the turning rate is $\alpha=1$.

We recap the discussion above by the following theorem.

Theorem 6.2 For every $N$ there exist $l$ tori, $T^{l-1} \times T^{1}$, parameter families of stationary solutions of the system of ODEs $(23,24)$. These stationary solutions consist of radial schools of fish separated by a repulsion zone of radius $a$ and forming schools of $k_{j}$ fish where $k_{j} \leq \frac{b}{a}$ where $b$ is the radius of the zone of attraction. The schools correspond to members of the fixed points of the isotropy groups

$$
\Sigma_{\mathbf{k}, m}=\left(S_{k_{1}} \times \cdots \times S_{k_{1}}\right)^{m} \rtimes Z_{m}
$$

forming $m$ clusters of $l$ radial schools, the total number of fish being $N=m\left(k_{1}+\cdots+k_{l}\right)$. The clusters are distributed as mth roots of unity and the parameters in the torus $T^{l-1}$ parametrize the distance between the subgroups of each cluster. The direction angles of the schools are also mth roots of unity and are locked to the position angles. The remaining parameter $\phi \in T^{1}$ is the parameter of the diagonal rotation on the $2 N$ torus of angles $T^{2 N}$.

Proof: Consider the system of equations

$$
\dot{v}_{k}=\frac{\alpha}{N^{2}} \sum_{j=1}^{N} v_{j} \sum_{j=1}^{N} \cos \left(\phi_{j}-\phi_{k}\right)-\alpha v_{k}
$$

$$
\begin{array}{r}
v_{k} \dot{\phi}_{k}=\frac{\alpha}{N^{2}} \sum_{j=1}^{N} v_{j} \sum_{j=1}^{N} \sin \left(\phi_{j}-\phi_{k}\right) \\
\dot{r}_{k}=v_{k} \cos \left(\phi_{k}-\theta_{k}\right)
\end{array}
$$

$$
r_{k} \dot{\theta}_{k}=v_{k} \sin \left(\phi_{k}-\theta_{k}\right)
$$


and let $\phi_{k}=\omega_{k}$, so $v_{k}=v_{k}^{0} e^{-\alpha t}$ as in the discussion of the stationary solution (29) in Section 4. If we lock the position angles to the direction angles $\theta_{k}=\phi_{k}$ then the latter system (31) reduces to

$$
\begin{aligned}
\dot{r}_{k} & =\alpha v_{k} \\
r_{k} \dot{\theta}_{k} & =0
\end{aligned}
$$

The solution of this system is the asymptotically stationary solution

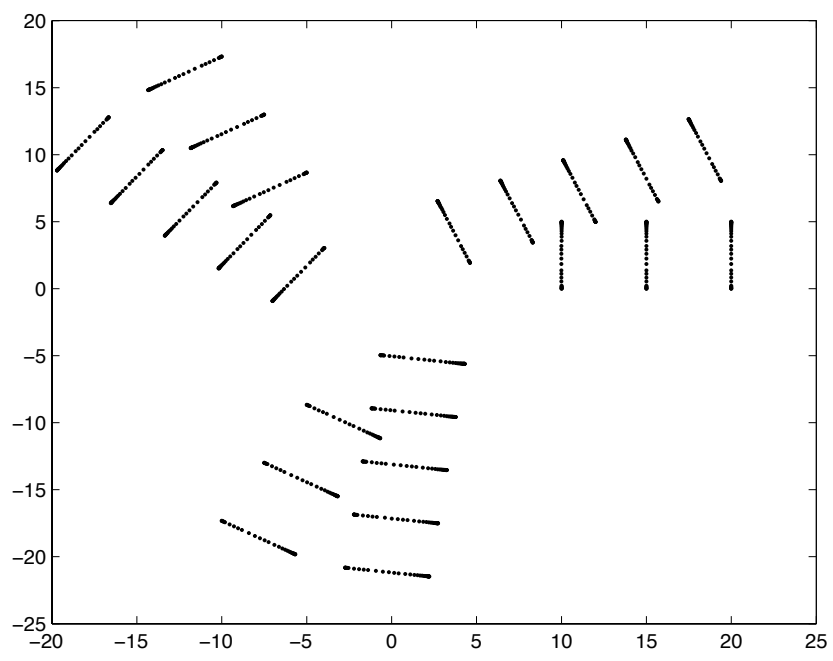

Figure 11: The Third Stationary School. This is a simulation of the ODEs finding the stationary solutions similar to those in Figure 8. The fish travel in straight lines, in three groups of two subschools each, one subschool having 3 fish the other one 5 fish, and stop at the stationary solutions, the turning rate is $\alpha=1$.

$$
r_{k}=r_{k}^{0}-\frac{v_{k}^{0}}{\alpha} e^{-\alpha t}, \theta_{k}=\omega_{k}
$$

If we restrict the initial conditions of the radii $r_{k}^{0}$ to have the symmetry of $S_{k_{j}}$ the permutations of $k_{j}$ objects, for each $j$, then the system (30) and the stationary solutions

$$
r_{k}=r_{k}^{0}, \quad \theta_{k}=\omega_{k}
$$

are invariant under the action of the isotropy subgroups $\Sigma_{\mathbf{k}, m}$ in Theorem 6.1, thus each subspace of fixed points Fix $\left[\Sigma_{\mathbf{k}, m}\right]$ is left invariant by the flow defined by the system. But this means that these subspaces are all the stationary solutions of the ODEs $(23,24)$ (and $(30,31)$ ) that possess these symmetries. The parametrization of these orbits by points on $T^{l-1} \times T^{1}$ was explained above. The zones of attraction and repulsion are explained in Section 9. The repulsive and attractive terms added to the equation in Section 9 are still invariant under the action of the isotropy subgroups $\Sigma_{\mathbf{k}, m}$ and they fix the distance between the fish in each subgroup of $k_{j}$ fish to be $a$, and the number $k_{j}$ to be the integer value of $b / a$.

QED 


\section{The Existence of Tori}

Dynamical tori have been observed for both numerical simulations and in observations of fish schools, see [28] and [5]. We now prove the existence of periodic orbits that can perturb into such tori, under deterministic perturbations, for two isotropy subgroups of stationary solutions.

Theorem 7.1 Assume that the alignment function has some higher order harmonic components

$$
f\left(\phi_{j}-\phi_{k}\right)=\sin \left(\phi_{j}-\phi_{k}\right)+\sum_{q=1} a_{q} \cos \left(q\left(\phi_{j}-\phi_{k}\right)\right)+b_{q} \sin \left(q\left(\phi_{j}-\phi_{k}\right)\right)
$$

Then the fixed point sets of the isotropy subgroups $\left(S_{k}\right)^{N} \rtimes Z_{m}, N=m k$, and $\left(S_{k_{1}} \times S_{k_{2}}\right)^{m} \rtimes Z_{m}, N=$ $\left(k_{1}+k_{2}\right) m$, generically contain periodic orbits (under rotation parametrized by $\phi$ ) of stationary solutions of the ODEs $(23,24)$.

Proof: The theorem is an application of the following theorem of Ashwin and Swift [3] to the ODEs $(23,24)$.

QED

Theorem 7.2 Every Fix $\left[\left(S_{k}\right)^{N} \rtimes Z_{m}\right], N=m k$, and Fix $\left[\left(S_{k_{1}} \times S_{k_{2}}\right)^{m} \rtimes Z_{m}\right], N=\left(k_{1}+k_{2}\right) m$, generically contain a periodic orbit with diagonal flow.

Theorem 7.2 is proven in Ashwin and Swift [3].

\section{Stability of Schools and Tori}

The stability of some of the configurations in Theorem 6.2 is worked out in the literature. In particular, the stability of $m$ circling clusters with a school of $k$ fish each $\left(S_{k}\right)^{m} \rtimes Z_{m}$, see Figure 6 , discussed above, is known. We start with the following result see Okuda [27] and Watanabe and Swift [41], that covered the $\left(S_{k}\right)^{m} \rtimes Z_{m}$ and $S_{N}$ cases respectively. The lemma also covers the stability of the dynamical tori whose existence was established in Section 7. Suppose that we have an alignment function $f$ that is $\sin \left(\phi_{j}-\phi_{k}\right)$ to leading order but is allowed to have higher order (in a small parameter) harmonic content

$$
\sum_{q=1} a_{q} \cos \left(q\left(\phi_{j}-\phi_{k}\right)\right)+b_{q} \sin \left(q\left(\phi_{j}-\phi_{k}\right)\right)
$$

Lemma 8.1 Let $N=m k$ and assume $(\bar{v}, \bar{\phi})$ is an $\left(S_{k}\right)^{m} \rtimes Z_{m}$ invariant stationary solution of (23) (with $f$ as above) or a periodic solution, when $f$ contains higher harmonics. Then the eigenvalues of the Jacobian linearized about $(\bar{v}, \bar{\phi})$ are:

1. $\lambda_{0}=0$; one eigenvalue corresponding to the one torus action (rotation)

2. $\lambda_{j}^{r}, \quad 1 \leq j \leq m-1, m-1$; rotation eigenvalues

3. $\lambda^{p}$; permutation eigenvalues with multiplicity $m(k-1)$ 
These eigenvalues are given by the formulas

$$
\lambda_{j}^{r}=\frac{v}{2 N}\left(\sum_{q \in \mathcal{M}(m)_{j}^{1}} q\left(b_{q}+i a_{q}\right)+\sum_{q \in \mathcal{M}(m)_{j}^{2}} q\left(b_{q}+i a_{q}\right)-2 \sum_{q \in \mathcal{M}(m)} q b_{q}\right)
$$

where

$$
\begin{array}{r}
\mathcal{M}(m)=\{m l \mid l=1,2, \ldots\} \\
\mathcal{M}(m)_{j}^{1}=\{m l-j \mid l=1,2, \ldots\} \\
\mathcal{M}(m)_{j}^{2}=\{m l+j \mid l=1,2, \ldots\}
\end{array}
$$

and

$$
\lambda^{p}=-\frac{v}{N} \sum_{q \in \mathcal{M}(m)} q b_{q}
$$

We also get $N$ eigenvalues equal to zero, corresponding to the $v_{k}$ directions.

The eigenvalues, first calculated in Okuda [27], are written in the form given in Brown, Holmes, and Moehlis [9]; the terminology "rotation" and "permutation" eigenvalues was first introduced in Ashwin and Swift [3].

We now examine the stability of the fish schools using Lemma 8.1. First consider $\left(S_{k}\right)^{m} \rtimes Z_{m}$ and consider the ODEs $(23,24)$. Then Lemma 8.1 gives the eigenvalues, see [9], $\lambda_{0}=0$, and

1. $\lambda^{p}=0$, with multiplicity $m(k-1)$

2. $\lambda_{j}^{r}=\left\{\begin{array}{l}\frac{v}{2 N}, \text { for } j=1 \text { and } m-1 \\ 0, \text { with multiplicity } m-3\end{array}\right.$

In addition, we get $N$ zero eigenvalues corresponding to the $v_{k}$ directions. The eigenvalues corresponding to the $r_{k}$ and $\theta_{k}$ coordinates are all zero. We conclude as in Section 5 that the $\left(S_{k}\right)^{m} \rtimes Z_{m}$ stationary solutions are unstable.

Next we add the higher harmonic content to the alignment function $f$. We only add one higher harmonic mode

$$
a_{q} \cos \left(q\left(\phi_{j}-\phi_{k}\right)\right)+b_{q} \sin \left(q\left(\phi_{j}-\phi_{k}\right)\right)
$$

Then Lemma 8.1 gives the eigenvalues (see [9]) $\lambda_{0}=0$, and

1. $\lambda^{p}=-\frac{v}{N} q b_{q}$, with multiplicity $m(k-1)$

2. $\lambda_{j}^{r}=\left\{\begin{array}{r}\frac{v}{2 n}\left(1-2 q b_{q}\right), \text { for } j=1 \text { and } m-1 \\ -\frac{v}{N} q b_{q}, \text { with multiplicity } m-3\end{array}\right.$

Thus if $b_{q}>\frac{1}{2 q}$ then the stationary solution $\left(S_{k}\right)^{m} \rtimes Z_{m}$ of $(23,24)$, are stable except for the $3 N$ marginal $v_{k}, r_{k}$ and $\theta_{k}$ directions and the single marginal (unstable) direction of rotation on the torus. The computation is similar for the tori in Section 7 and they are also stable except for the $v_{k}, r_{k}$ and $\theta_{k}$ directions were we still get all eigenvalues zero. 
Now let us consider the migratory school $S_{N}$. Then there are no rotation eigenvalues and Lemma 8.1 gives the eigenvalues

$$
\lambda^{p}=-\frac{v}{N}
$$

with multiplicity $N-1$. The $v_{k}$ directions give $N$ zero eigenvalues. The $r_{k}$ and $\theta_{k}$ directions give $N$ negative eigenvalues $-\frac{v}{r_{k}^{0}+v t}$, for every fixed $t$. We have linearized about the linear orbit as in Section 5. This means that apart from the speeds $v_{k}$, the migrating schools are stable as we saw in Section 5, except for the marginal direction of the drift in the direction angle $\phi$ of the school, with the eigenvalue $\lambda_{0}=0$.

\subsection{Structurally Stable Heteroclinic Orbits}

Structurally stable heteroclinic cycles exist in the phase space of the system (23) see Ashwin and Swift [3]. This is unusual for ODE systems because usually such heteroclinic cycles are structurally unstable. However, in the presence of symmetries these cycles may be stable which means that they persist when small perturbations are added to ODE's. The configuration possessing the structurally heteroclinic cycles (under the diagonal flow parametrized by $\phi$ ) corresponds to fixed points of the isotropy subgroup

$$
S_{N / 2} \times S_{p} \times S_{q}, \quad p+q=\frac{N}{2}
$$

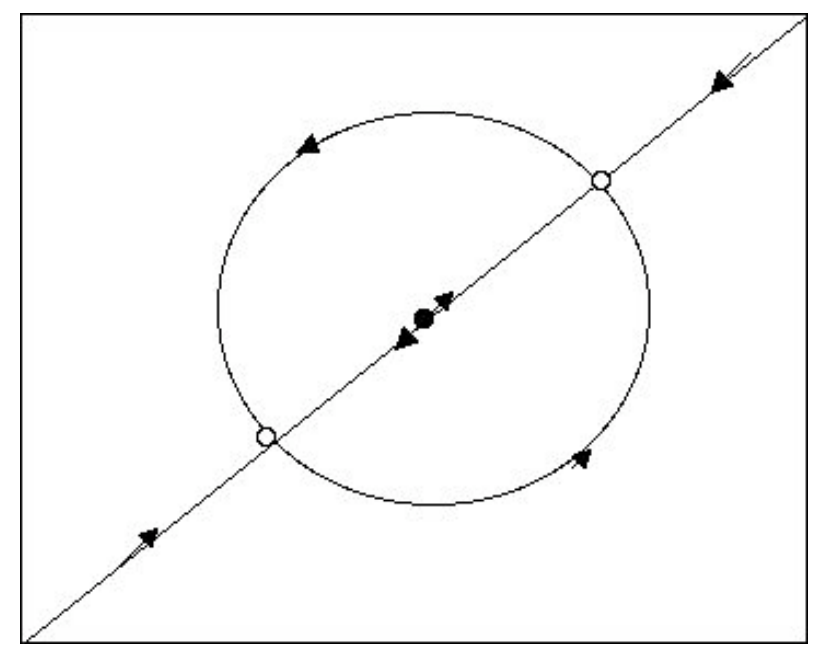

Figure 12: The structurally stable heteroclinic orbits. This figure is a cartoon of the heteroclinic orbits based on Figure 11 in Ashwin and Swift [3]. The square is the canonical invariant region for the subspaces of isotropy subgroups. The two circles are the two $S_{N / 2} \times S_{N / 2}$ stationary solutions. The dark circle is an unstable (under the phase flow) stationary solution and the two heteroclinic cycles consist of $S_{N / 2} \times S_{p} \times S_{q}$ stationary solutions.

where $N$ is the total number of fish. This corresponds to three radial schools of fish, with $N / 2$, $p$ and $q$ members respectively. The heteroclinic orbits can be illustrated on a canonical invariant region, see [3], illustrated in Figure 12. The two stable heteroclinic cycles shown in the figure are 
the connections between two solutions with isotropy $S_{N / 2} \times S_{N / 2}$, or $q=0$. By letting $v_{k}=0$ and imposing the same symmetry on the $r$ and $\theta$ coordinates as in Theorem 6.2, we get heteroclinic connections between stationary solutions corresponding to the last isotropy subgroups, for the whole system $(23,24)$. This corresponds to a school of fish containing three subschools of $N / 2, p$ and $q$ fish respectively, moving from one such a stationary school, consisting of two subschools of $N / 2$ each, to another such school. The most likely scenario for this is that one subschool of $N / 2$ fish splits into two subschools of $p$ and $q$ fish each and subsequently these two subschools merge again.

\section{Repulsion and Attraction}

We follow Couzin et al [10] in adding a zone of repulsion of radius $a$, a zone of orientation of radius $o$ and a zone of attraction of radius $b$ center at every fish, see Figure 13.

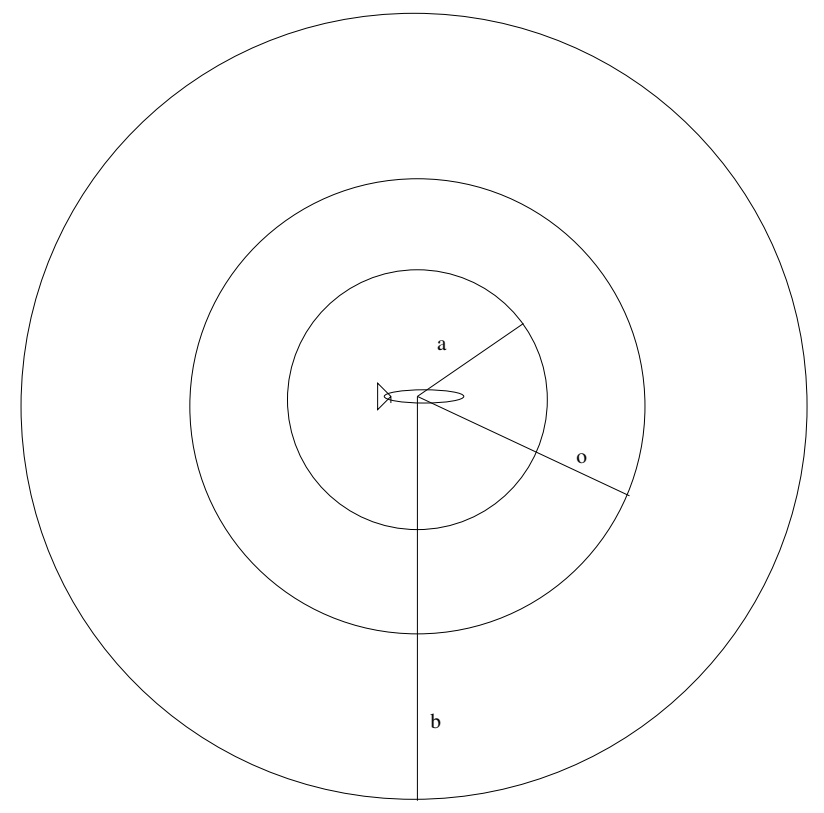

Figure 13: The zones of repulsion, orientation and attraction

We add repulsive and attractive terms to the ODEs resulting in the equations

$$
\dot{v}_{k}=\frac{\alpha}{N^{2}} \sum_{j=1}^{N} v_{j} \sum_{j=1}^{N} \cos \left(\phi_{j}-\phi_{k}\right)-\alpha v_{k}+\sum_{j \neq k} g_{k j} \frac{r_{k}-r_{j} \cos \left(\theta_{j}-\theta_{k}\right)}{\left(r_{k}^{2}+r_{j}^{2}-2 r_{k} r_{j} \cos \left(\theta_{j}-\theta_{k}\right)\right)^{1 / 2}}
$$

$$
\begin{gathered}
v_{k} \dot{\phi}_{k}=\frac{\alpha}{N^{2}} \sum_{j=1}^{N} v_{j} \sum_{j=1}^{N} \sin \left(\phi_{j}-\phi_{k}\right)-\sum_{j \neq k} g_{k j} \frac{r_{j} \sin \left(\theta_{j}-\theta_{k}\right)}{\left(r_{k}^{2}+r_{j}^{2}-2 r_{k} r_{j} \cos \left(\theta_{j}-\theta_{k}\right)\right)^{1 / 2}} \\
\dot{r}_{k}=v_{k} \cos \left(\phi_{k}-\theta_{j}\right)
\end{gathered}
$$

$$
r_{k} \dot{\theta}_{k}=v_{k} \sin \left(\phi_{k}-\theta_{j}\right)
$$


The function $g_{k j}=g\left(\left|r_{k}-r_{j}\right|\right)$ is 1 when $\left|r_{k}-r_{j}\right| \leq a$, 0 when $a<\left|r_{k}-r_{j}\right|<o$ and -1 when $o<\left|r_{k}-r_{j}\right| \leq b$. In this paper, we assume for the sake of the stability analysis that $g_{k j}$ is a smooth function, $a=o$ and $g\left(\left|r_{k}-r_{j}\right|\right)=0$ when $\left|r_{k}-r_{j}\right|=a$. This means that if $g=\frac{\partial G}{\partial r}$, then the potential $G$ has a single minimum at $r=a$. This will stabilize the $r_{k}$ directions in the stability analysis in the previous sections. In fact the configurations in Theorems 6.2 and 7.1 that are stable in $\theta$, will have local minima when the individual fish are separated by the distance $a$, and so these configurations are stable. We also want the field of vision and the sensitivity of the lateral line of the fish to be limited so we set $\alpha=0$ if $\left|r_{k}-r_{j}\right|>b$.

There are obviously many other ways of modeling the repulsion and attraction of the fish, see for example [17], but the terms above have the advantage of preserving the group invariance and therefore making the analysis in the previous sections still apply. There does not seem to be any biological reason to pick one form of the repulsion or attraction terms over another as long as they make the fish turn toward or away from each other in a smooth fashion. This changes when the number of fish increases and one see drastically different behavior depending on the attraction and repulsion as $N \rightarrow \infty$, see [17]. In simulations of the discrete model (1) the direction angle of the fish within the zone of repulsion is typically computed and it averaged with all the angles with a negative sign. This makes fish turn away from fish that are too close. For the fish in the attraction zone the direction angle is computed and it averaged with all the angles but with a positive sign. This makes the fish turn toward a fish in the attraction zone.

\section{Deterministic Perturbations}

The ODE system that we simulate to compare with the simulations of the discrete system and to include small environmental effect is

$$
\begin{gathered}
\dot{v}_{k}=\frac{\alpha}{N^{2}} \sum_{j=1}^{N} v_{j} \sum_{j=1}^{N} \cos \left(\phi_{j}-\phi_{k}\right)-\alpha v_{k}+v \\
v_{k} \dot{\phi}_{k}=\frac{\alpha}{N^{2}} \sum_{j=1}^{N} v_{j} \sum_{j=1}^{N} \sin \left(\phi_{j}-\phi_{k}\right)+v_{k} \omega \\
\dot{r}_{k}=v_{k} \cos \left(\phi_{k}-\theta_{k}\right) \\
r_{k} \dot{\theta}_{k}=v_{k} \sin \left(\phi_{k}-\theta_{k}\right)
\end{gathered}
$$

where $v$ and $\omega$ are deterministic perturbation.

We do not expect this system to tell us much about the migratory solutions but it should give information about what the schools of fish close to the stationary solutions in Theorem 6.2 are doing. In particular we can use the solutions of this system as a guide to find special solutions of the discrete system (1), such as periodic solutions and tori and examine their stability.

Indeed the system $(35,36)$ has periodic orbits close to the stationary solutions in Theorem 6.2 , see Figures 8,9 and 10 and they are unstable. Tori also exist but these will be discussed in another publication see [5]. 
We will now discuss what influence the stationary solutions in Section 6-7 can be expected to have on the simulations of the school of fish. In simulations of the discrete model (1) with small amount of noise added three types of school behaviors are observed, that can be characterized as migratory, swarming and circulating. It is reasonable to expect small perturbations of the stationary solutions to lead to circling solutions and those to turn into swarms when the noise level is increased. In Barbaro, Birnir and Taylor [5] simulations of the discrete system are compared to simulations of the ODEs. Perturbations of the ODEs do in fact lead to metastable periodic orbits and their instabilities are explored in [5]. It is also shown in this paper that the ODE system is capable of producing its own noise.

An ODE model that includes the zones of attraction and repulsion as well as environmental effects can be written in the form

$$
\begin{aligned}
v_{k} \dot{\phi}_{k}=\frac{\alpha}{N^{2}} \sum_{j=1}^{N} v_{j} \sum_{j=1}^{N} \sin \left(\phi_{j}-\phi_{k}\right)- & \sum_{j \neq k} g_{k j} \frac{r_{j} \sin \left(\theta_{j}-\theta_{k}\right)}{\left(r_{k}^{2}+r_{j}^{2}-2 r_{k} r_{j} \cos \left(\theta_{j}-\theta_{k}\right)\right)^{1 / 2}}+\frac{1}{v_{k}} \frac{\partial}{\partial \phi_{k}} G \\
\dot{r}_{k} & \left.=v_{k} \cos \left(\phi_{k}-\theta_{j}\right)\right) \\
r_{k} \dot{\theta}_{k} & =v_{k} \sin \left(\phi_{k}-\theta_{j}\right)
\end{aligned}
$$

where $G$ is a function modeling the influence of the environment on the direction and speed of the school, and the function $g_{k j}$ hs the same values in zones of repulsion, orientation and attraction as above.

\section{The Case of the Capelin}

The capelin (Mallotus villosus) is a pelagic species of fish that undertakes extensive feeding and spawning migrations, covering distances of several hundred miles, see Vilhjálmsson [37] in the North Atlantic. The timing and the route of the migration, see Figure 14, are influenced by a variety of factors: environmental conditions such as boundaries between warm and cold water masses, certain isotherms which, as a rule, the fish do not cross, bottom topography, oceanic currents, density of food and internal variables such as the physiological state and the maturity of each fish, see [37, 20].

The migration of the capelin shown in Figure 14 proceeds as follows. The spawning grounds are off the southwest coast of Iceland. The mature stock migrates northwards in early summer to feeding grounds in the Jan Mayen area. Jan Mayen is the island close to the top right corner of the upper map in Figure 14. There the capelin stock takes advantage of the enormous increase in the biomass of the zooplankton in the arctic summer. In early fall, the stock returns to the north and northwest coast of Iceland. It gradually moves clockwise around the island and ends up at the spawning grounds of the southwest coast in March to April. During some years, a component of the stock migrates in a counterclockwise direction to the spawning grounds, the size of this component being highly variable, see $[37,20]$. 

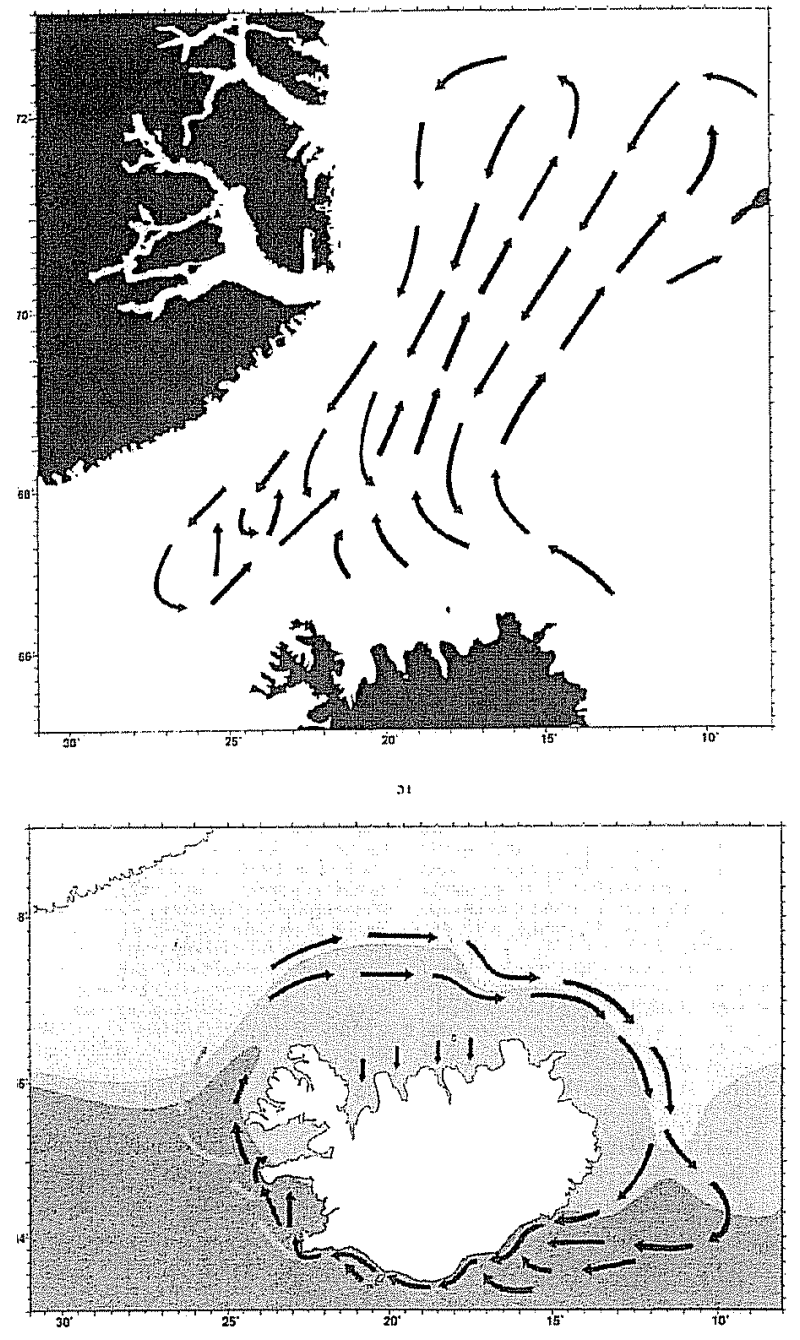

Figure 14: The Migration of the Capelin, from H. Vilhjálmsson [37]. The top figure shows the feeding migration taking place in summer and early fall. The bottom figure shows the spawning migration taking place in winter. 
The capelin migration was roughly captured by the simulation of Hubbard, Babak, Magnússon and Sigurdsson [20] and in much more detail by Einarsson, Magnússon and Sigurdsson [26]. However, both of these simulations relied on oriented noise to pull the stock to the feeding and spawning grounds. The question one must ask with the insight into the dynamics of the solutions in particular the migratory (28) and circling (29) solutions, provided by the ODEs, is: can the migration be constructed solely from these solutions and the environmental factors? The environmental factor are largely known and can be built into the model using the function $G$ in (37). The currents and overall variations in food and temperature are tabulated for past years as functions of time and can be forcasted to some extent for future years. This question is currently being addressed in simulations. One does not expect any simplistic solutions such as Iceland being the center of a circling solution. Rather the migrations would be a patchwork of migratory and circling solutions, the latter turning into swarms when the noise level is sufficiently high. In particular, it remains to determine how the schools can switch from one phase (say migratory) to another (circling) and what triggers this transition.

\section{Conclusion}

The ODE model for the dynamics of schools of fish derived from the discrete model (1) is a useful tool to examine the dynamics of fish schools. We have seen that the ODE model possesses a wide variety of solutions that can be associated with schools of fish and their dynamics. All symmetric stationary and circling solutions can be described by equivariant bifurcation theory and their stability found. The migratory solutions are stable whereas the circling solutions are unstable. The ODEs provide the tools for understanding the structure of the phase space of the schools of fish but much work remains to be done to fully understand the global structure. Finally, we can use the ODE to find corresponding structures in the phase space of the discrete system (1), see [5].

Perhaps the most important lesson learned from the simulations of the ODEs is a paradigm shift. Instead of looking for the biological reasons that drive the two different migratory phases the ODEs show us that these phases are already there. What is needed instead are the biological reason that induce a transition from one phase to another.

It also still remains to simulate a truly realistic school containing up to 1 million individuals. The simulations so far, being of such a smaller magnitude than the true number of fish in a typical school, must be interpreted as being of super-individuals where each point represents a school. This amounts to a course-graining of the problem and then all the schools in Sections 6-8 can be collapsed to a zone of (no) repulsion with radius $a=0$, see Section 9.

We exploited that the Kuromoto system [22, 23] of coupled oscillators sits in the ODE system $(23$, 24) describing the schools. The symmetries that we used in the equivariant bifurcation theory are those of the Kuromoto system. This raises the question whether the complex Kuromoto solutions for a density of oscillators, see [22], can also be applied to construct schools of fish for the limit when the number of individuals goes to infinity. The answer is yes, but this will be explored in another publications, see Birnir, Bonilla and Soler [6]. The stability of such solutions is also being explored numerically, see Birnir, Einarsson and Sigurdsson [7]. Boilla, Neu and Spigler [8], Crawford and Davis [11, 12], Watanabe and Strogatz [40, 32] and Smereka [31] can be consulted for information on the Kuromoto and related systems as the number of individuals goes to infinity. Based on the simulations of Smereka [31], we expect to see several complex states (phases) strongly influenced by noise. It seems that in order to fully understand schools of fish and their 
dynamics, one must understand these complex states of the incoherent noise-driven Kuromoto system. This remains mostly an open problem.

Acknowledgments The author was supported by grants number DMS-0352563 from the National Science Foundation whose support is gratefully acknowledged. He also want to thank the late Kjartan Magnússon and Sven Sigurdsson at the University of Iceland for useful discussions. He is indebted to his students Alethea Barbaro and Baldvin Einarsson for reading the manuscript and correcting it. The simulations were done by Alethea Barbaro. The author also wants to thank Andrea Bertozzi and her students and Chad Topaz at UCLA, Allison Kolpas and Jeff Moehlis at UCSB and Daniel Grunbaum at the University of Washington for helpful suggestions. Some of the simulations are being done on a cluster of workstations, funded by a National Science Foundation SCREMS grant number DMS-0112388.

\section{A The ODEs in Cartesian Coordinates}

In this appendix we express the initial value problem (23) and (24) in Cartesian coordinates.

In complex coordinates the initial value problem is

$$
\ddot{z}_{k}+\alpha \dot{z}_{k}=\frac{\alpha}{N^{2}} \sum_{j=1}^{N}\left|\dot{z}_{j}\right| \sum_{j=1}^{N} e^{i \arg \left(\dot{z}_{j}\right)}
$$

with the initial positions and velocities

$$
z_{k}(0)=z_{k}^{0}, \quad \dot{z}_{k}(0)=\dot{z}_{k}^{0}
$$

where $\alpha$ is a unitless turning rate or inertia, for $1 \leq k \leq N$.

The real initial value problem is

$$
\begin{aligned}
& \ddot{x}_{k}+\alpha \dot{x}_{k}=\frac{\alpha}{N^{2}} \sum_{j=1}^{N} v_{j} \sum_{j=1}^{N} e^{i \phi_{j}} \\
& \ddot{y}_{k}+\alpha \dot{y}_{k}=\frac{\alpha}{N^{2}} \sum_{j=1}^{N} v_{j} \sum_{j=1}^{N} e^{i \phi_{j}}
\end{aligned}
$$

with the initial positions and velocities

$$
\begin{aligned}
& x_{k}(0)=x_{k}^{0}, \quad \dot{x}_{k}(0)=\dot{x}_{k}^{0} \\
& y_{k}(0)=y_{k}^{0}, \quad \dot{y}_{k}(0)=\dot{y}_{k}^{0}
\end{aligned}
$$

where again, $\alpha$ is a unitless turning rate. Here

$$
v_{k}=\sqrt{\dot{x}_{k}^{2}+\dot{y}_{k}^{2}}
$$

and

$$
\phi_{k}=\tan ^{-1}\left(\frac{\dot{y}_{k}}{\dot{x}_{k}}\right)
$$

The whole system must be solved for every $k$ such that $1 \leq k \leq N$. 


\section{References}

[1] I. Aoki. A simulation study on the schooling mechanism in fish. Bull. Jap. Soc. Sci. Fish., 48:1081-1088, 1982.

[2] V. Arnold. Ordinary Differential Equations. MIT Press, Boston, 1973.

[3] P. Ashwin and J. Swift. The dynamics of n weakly coupled identical oscillators. J. Nonlin. Sci., 2:69-108, 1992.

[4] P. Babak, K. G. Magnússon, and S. Th. Sigurdsson. Dynamics of group formation in collective motion of organisms. Math. Med. Biol., 21:269-292, 2004.

[5] A. Barbaro, B. Birnir, and K. Taylor. A comparison of simulations of discrete and ode models for the motion of pelagic fish. UCSB Preprint, 2006.

[6] B. Birnir, L. Bonilla, and J. Soler. Complex fish schools. UCSB Preprint, 2006.

[7] B. Birnir, B. Einarsson, and S. Sigurdsson. Simulations and stability of complex fish schools. RH Preprint, 2006.

[8] L. L. Bonilla, J. C. Neu, and R. Spigler. Nonlinear stability of incoherence and collective synchronization in a population of coupled oscillators. J. Stat. Phys., 67:313-330, 1992.

[9] E. Brown, P. Holmes, and J. Moehlis. Globally coupled oscillator networks. In Perspective and Problems in Nonlinear Science, A celebratory Volume in Honor of Lawrence Sirovich, eds. E. Kaplan, J. E. Marsden and K. R. Sreenivasan, pages 183-215, 2003.

[10] J. Krause Couzin, I.D., R. James, G.D. Ruxton, and N.R. Franks. Collective memory and spatial sorting in animal groups. J. theor. Biol., 218:1-11, 2002.

[11] J. Crawford. Scaling and singularities in the entrainment of globally coupled oscillators. Phys. Rev. Lett., 74:4341-4344, 1995.

[12] J. Crawford and K. Davis. Synchronization for globally coupled phase oscillators: singularities and scalings for general couplings. Physica D, 125(1-2):1-46, 1999.

[13] A. Czirók, H.E. Stanley, and T. Vicsek. Spontaneously ordered motion of self-propelled particles. J. Phys. A: Math. Gen., 30:1375-1385, 1997.

[14] A. Czirók, M. Vicsek, and T. Vicsek. Collective motion of organisms in three dimensions. Physica A, 264:299-304, 1999.

[15] A. Czirók and T. Vicsek. Collective behavior of interacting self-propelled particles. Physica A, 281:17-29, 2000.

[16] A. Czirók and T. Vicsek. Collective Motion in Fluctuations and Scaling in Biology, ed. T. Vicsek, chapter 6, pages 177-209. Oxford University Press, Oxford, UK, 2001.

[17] M. R. D’Orsogna, Y. L. Chuang, A. L. Bertozzi, and L. Chayes. Self-propelled particles with soft-core interactions: patterns, stability and collapse. Phys. Rev. Lett., 96:104302, 2006. 
[18] M. Golubitsky, I. Stewart, and D. Schaeffer. Singularities and Groups in Bifurcation Theory, Vol. 2. Springer, New York, 1988.

[19] F. H. Heppner. Three-dimensional structure and dynamics of bird flocks. In animal groups in three dimensions, eds J. K. Parrish and W. E. Pamner, Cambridge University Press, 1997.

[20] S. Hubbard, S. Sigurdsson P. Babak, and K. Magnússon. A model of the formation of fish schools and migrations of fish. Ecological Modelling, 174:359-374, 2004.

[21] A. Huth and C. Wissel. The simulation of the movement of fish schools. J. Theor. Biol., 156:3565-385, 1992.

[22] Y. Kuramoto. Self-entrainment of a population of coupled nonlinear oscillators. Proceedings of the International Symposium on Mathematical Problems in Theoretical Physics, ed. H. Araki, Lecture Notes in Physics, 39:420-422, 1975.

[23] Y. Kuramoto. Chemical Oscillations, Waves and Turbulence. Springer, New York, 1984.

[24] K. G. Magnússon, S. Sigurdsson, P. Babak, S. F. Gudmundsson, and E. H. Dereksdóttir. A continous density kolmogorov type model for a migrating fish stock. Discr. Cont. Dyn. Sys., B 4(3):695-704, 2004.

[25] K. G. Magnússon, S. Sigurdsson, and E. H. Dereksdóttir. A simulation model for the capelin migrations in the north atlantic. Nonlinear Analysis: Real World Applications, 6:747-771, 2005.

[26] K. G. Magnússon, S. Th. Sigurdsson, and B. Einarsson. A discrete and stochastic simulation model for migration of fish with application to capelin in the seas around iceland. Technical Report RH-20-04, Science Institute, University of Iceland, 2004.

[27] K. Okuda. Variety and generality of clustering in globally coupled oscillators. Physica D, 63:424-436, 1993.

[28] B. L. Partridge. The structure and function of fish schools. Sci. Am., 245:90-99, 1982.

[29] B. L. Partridge and T. J. Pitcher. The sensory basis of fish schools: relative role of lateral lines and vision. Journ. Compar. Physiol., 135:315-325, 1980.

[30] C. W. Reynolds. Flocks, herds and schools: a distributive behavioral model. Comput. Graph., 21:24-34, 1987.

[31] P. Smereka. Synchronization and relaxation for a class of globally coupled hamiltonian systems. Physica D, 124:104-125, 1998.

[32] S. Strogatz. From kuromoto to crawford: Exploring the onset of synchronization in populations of coupled oscillators. Physica D, 143:1-20, 2000.

[33] J. Toner and Y. Tu. Long range order in a two-dimensional xy model: how birds fly together. Phys. Rev. Lett., 73:1375-1385, 1995.

[34] J. Toner and Y. Tu. Flocks, herds and school: a quantative theory of flocking. Phys. Rev. E, 58:4828, 1998. 
[35] T. Vicsek, A. Czirók, E. Ben-Jacob, I. Cohen, and O. Shochet. Novel type of phase transition in a system of self-driven particles. Physical Review Letters, 75(6):1226-1229, 1995.

[36] T. Vicsek, A. Czirók, I.J. Farkas, and D. Helbing. Application of statistical mechanics to collective motion in biology. Physica A, 274:182-189, 1999.

[37] H. Vilhjálmsson. The icelandic capelin stock. Journ. Marine Research Inst. Reykjavík, XIII(2):281, 1994.

[38] H. Vilhjálmsson. Capelin (mallotus villosus) in the Iceland-East Greenland-Jan Mayen ecosystem. ICES Journal of Marine Science, 59:870-883, 2002. Available online at http//www.idealibrary.com, doi:10.1006/jms.2002.1233.

[39] H. Vilhjálmsson and J. E. Carscadden. Assessment surveys for capelin in the Iceland-East Greenland-Jan Mayen area, 1978-2001. ICES Journal of Marine Science, 59:1096-1104, 2002. Available online at http//www.idealibrary.com, doi:10.1006/jms.2002.1232.

[40] S. Watanabe and S. Strogatz. Constant of the motion for superconducting josephson arrays. Physica D, 74:195-253, 1994.

[41] S. Watanabe and J. Swift. Stability of periodic solutions in series arrays of josephson junctions with internal capacitance. J. Nonl. Sci., 7:503-536, 1997. 